Audiology

Neurotology
Audiol Neurotol 2010;15:155-167

DOI: $\underline{10.1159 / 000241096}$
Received: October 24, 2008

Accepted after revision: June 12, 2009

Published online: September 24, 2009

\title{
Change in Cochlear Response in an Animal Model of Otitis Media with Effusion
}

Chenkai Dai Rong Z. Gan

University of Oklahoma, Norman, Okla., USA

\section{Key Words}

Otitis media with effusion $\cdot$ Basilar membrane $\cdot$ Cochlear response

\begin{abstract}
Our previous studies confirm that middle ear mobility is reduced in the presence of otitis media with effusion (OME). Variations in middle ear function may result in changes in cochlear response in OME ears. With the long-term goal of evaluating cochlear function in OME ears, the aim of this study was to measure the displacement of the basilar membrane (BM) in guinea pig ears with OME. Vibrations of the BM at the apex and basal turn were measured in an in vitro preparation extracted 3 and 14 days after injection of lipopolysaccharide in the middle ear of guinea pigs. The results show that the displacement sensitivity of the BM at the apex and the basal turn to sound pressure in the ear canal was reduced up to $25 \mathrm{~dB}$ at their characteristic frequencies, respectively. Cochlear gain with respect to umbo movement was also changed in ears with OME in both groups. This study provides data for analysis of the change of $\mathrm{BM}$ vibration in a guinea pig OME model.

Copyright $\odot 2009$ S. Karger AG, Basel
\end{abstract}

\section{Introduction}

Otitis media with effusion (OME) is characterized by a nonpurulent effusion of the middle ear and causes most conductive hearing loss seen in the pediatric population. The reduction in umbo or stapes movement by middle ear fluid has been reported recently in human temporal bones [Ravicz et al., 2004; Gan et al., 2006; Dai et al., 2007, 2008] and guinea pigs [Dai and Gan, 2008]. The reduction in middle ear movement suggests that the cochlear mechanics could also be affected since the vibration of the stapes footplate serves as the input for the movement of cochlear fluid and the basilar membrane (BM). Moreover, the effusion interferes with the movement of the round window membrane, which may reduce BM movement by increasing cochlear impedance [Lynch et al., 1982].

Mechanical movement of the BM is the stimulus for hair cell and auditory nerve responses to the sound in the ear canal. Studies on variation of BM vibration in $\mathrm{OME}$ ears may reveal the correlation between the OME, a typical middle ear disease, and the change in cochlear function. Understanding this correlation may bring new insight into detection of OME with evaluation of cochlear function.

Most of our knowledge of cochlear mechanics is based on observations of BM motion at the basal turn and apex of the cochlea of animals including the guinea pig, the

Prof. Rong Z. Gan, PhD

School of Aerospace and Mechanical Engineering and Bioengineering Center

University of Oklahoma, 865 Asp Avenue, Room 200

Norman, OK 73019 (USA)

Tel. +1 405325 1099, Fax +1 405325 1088, E-Mail rgan@ou.edu 
gerbil and other mammals. Advanced laser Doppler interferometry has been used to measure the vibration of the $\mathrm{BM}$ at the characteristic frequency in live animals [Cody and Johnstone, 1980; Nuttall et al., 1991] and in isolated temporal bones [Ulfendahl et al., 1989; Gummer et al., 1993; Hemmert et al., 2000; International Team for Ear Research, 1989; Morioka et al., 1995]. In vitro measurements are useful because they permit relatively unobstructed access to many structures of the organ of Corti [Ulfendahl, 1997] and therefore can provide crucial information about cochlear mechanics.

In this study, we induced OME in guinea pigs via lipopolysaccharide (LPS) injection into the middle ear [Ohashi et al., 1991] and measured subsequent changes in the movement of the BM and tympanic membrane (TM) with a laser Doppler vibrometer. The changes observed in BM mobility at the basal turn and apex in OME ears suggest that OME may directly affect cochlear function through changing the ratio of stapes to umbo velocity over a frequency range of $200 \mathrm{~Hz}$ to $40 \mathrm{kHz}$. This study provides new information about the changes of cochlear mechanical response in OME ears.

\section{Methods}

\section{Experimental Protocol}

This study protocol was approved by the Institutional Animal Care and Use Committee of the University of Oklahoma and met the guidelines for the National Institutes of Health. Twelve Hartley guinea pigs ( 5 males, 7 females, average weight $300 \mathrm{~g}$ ), free from middle ear disease as evaluated by otoscopic examination, were used as experimental subjects. Another 3 animals (6 ears) were used as blank controls.

The OME model was created by injection of LPS into the middle ear of the guinea pig. LPS in saline $(100 \mu \mathrm{g} / \mathrm{ml}$, of Klebsiella pneumoniae, Sigma) was injected into the left ear through the ear canal skin beneath the edge of TM annulus using a $1-\mathrm{cm}^{3}$ syringe with a 26 -gauge needle under an operating microscope. The opposite right ear without injection served as a contralateral control. Injection into one ear is not expected to affect the opposite ear [Ar et al., 2007]. The injected animals were divided into two groups based on survival time after LPS injection (group 1: 3 days, and group 2: 14 days). Each group contained 6 guinea pigs. These times reflect early and chronic stages, respectively, of OME after LPS injection [Ohashi et al., 1991].

\section{Confirmation of OME}

Three and 14 days after LPS injection, the animals were anesthetized with an intramuscular injection of ketamine $(40 \mathrm{mg} / \mathrm{kg})$ and the TM examined with an otoscope to determine the color and position of the TM, as well as the presence of middle ear fluid. Middle ear compliance and pressure were then measured using a multiple-frequency tympanometer (Maico MI24, Minn., USA). The volume of fluid in the middle ear was also measured once transfer function measurements were finished, by opening the temporal bone through the bulla and withdrawing the effusion into a syringe.

The criteria for OME, or possible experimental findings consistent with OME, included the presence of: (1) a dark and opaque TM as determined by otoscopy; (2) low compliance and negative middle ear pressure as detected by tympanometry, and (3) a volume of middle ear fluid $>0.1 \mathrm{ml}$.

\section{Guinea Pig Temporal Bone Preparation}

On the end day of the postinoculation period, the guinea pig was anesthetized (ketamine, $100 \mathrm{mg} / \mathrm{kg}$, i.m. and xylazine, $10 \mathrm{mg} /$ $\mathrm{kg}$, i.m.) and decapitated. The temporal bone was quickly removed. A reflective tape $(0.05 \mathrm{mg})$ with a diameter of $0.5 \mathrm{~mm}$ was placed in the center of the lateral surface of the TM (umbo) to be used as a laser target.

A small hole with diameter of 1-2 mm was made on the posterior wall of the bulla to expose the cochlea. The cochlea was opened by carefully thinning the bony wall with a diamond drill bit over the scala tympani of the basal turn (the first turn) and the scala vestibuli of the apical region (fourth turn). The cochleostomy was between 0.2 and $0.4 \mathrm{~mm}$ in diameter and a minimal amount of perilymph escaped the scala tympani. Three or 4 solidglass microbeads (30 $\mu \mathrm{m}$ diameter, Mo-Sci Corp., Rolla, Mo., USA) were placed on the BM to act as light-reflecting objects. At the apex, Reissner's membrane was carefully removed with an instrument with a sharp tip under a microscope and the beads were placed carefully on the organ of Corti as shown in figure $1 \mathrm{~b} . \mathrm{Be}-$ cause of the high density of the glass beads compared with cochlear fluid, the beads do not float but sit on the BM. The holes made on the cochlea and bulla were sealed with glass sheets. The position of the bulla was adjusted to secure the position of the cochlea relatively high to maintain the effusion inside the bulla and in contact with the round window. If the bulla was completely filled with fluid, a small amount of fluid was removed to expose the cochlea. It is noted that any middle ear pressure differences were equalized with atmospheric pressure because of the opening of the bulla.

All vibration measurements were completed within $2 \mathrm{~h}$ of the guinea pigs' deaths. The measurement of BM movement was conducted on isolated temporal bones and assumed to be passive cochlear mechanical measurements. However, it is noted that the measurements were finished promptly after death and that the vitality of hair cells in the BM was unknown. The main purpose of this study was to determine the difference in BM movement between control and LPS-treated ears.

\section{Laser Doppler Interferometry}

The temporal bone was wrapped in gauze wetted with saline to prevent desiccation of the specimen during the experiment. The vibration of the TM at the umbo and the BM at the apex and basal turn in each temporal bone was then measured. The LPStreated ear was evaluated first, followed by the untreated ear in each animal. This order was chosen to avoid any time-dependent effect on vibration of the TM or BM. After extensive training and practice, we were able to complete vibration measurements within $2 \mathrm{~h}$ after the removal of the guinea pigs' heads.

The diagram in figure la shows the setup with the laser Doppler vibrometer, which has been described elsewhere [Dai and Gan, 2008]. Briefly, 80-dB-SPL pure tones within the fre- 
Fig. 1. a Schematic of the experimental setup with the laser directed to the BM at the basal turn and the organ of Corti at the apex through the cochleostomy (b). Mic = Microphone.

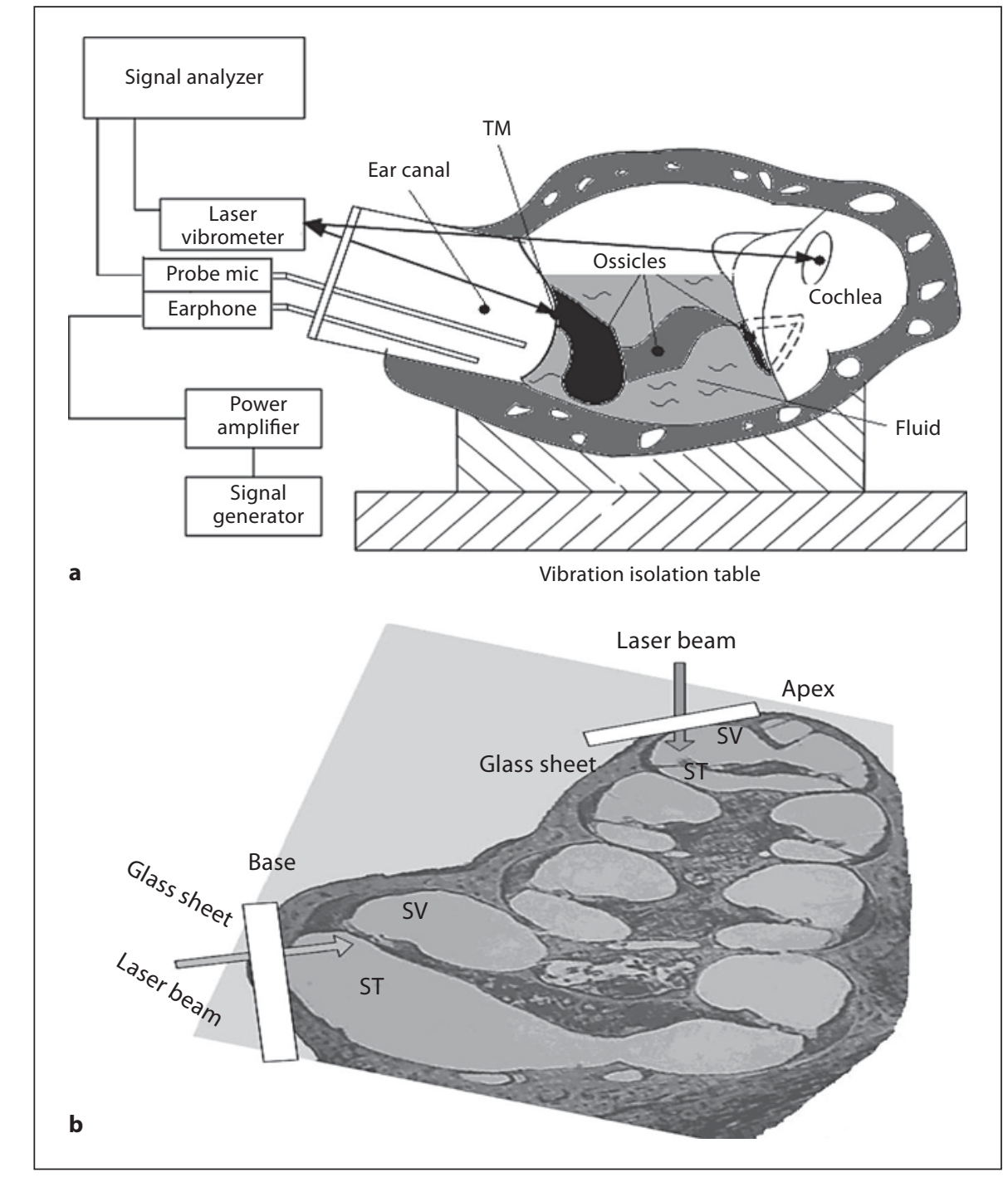

quency range of $200 \mathrm{~Hz}-40 \mathrm{kHz}$ from a function generator (HP $35670 \mathrm{~A}$ ) were presented to the ear canal near the umbo by an insert earphone. The input sound included 80 spectral lines at 80 $\mathrm{dB}$ SPL each. A transparent hard plastic tube (diameter of $4 \mathrm{~mm}$ ) as the sound delivery tube was placed in the ear canal and sealed to the canal wall with dental cement around the bony rim of the ear canal. A probe microphone (B\&K 4938, Denmark, frequency up to $100 \mathrm{kHz}$ ) was used for monitoring the input SPL. The probe tubes of the microphone and earphone were integrated into the sound delivery tube and the tip of the probe tube was positioned approximately $2 \mathrm{~mm}$ from the umbo. The laser beam was focused on the TM target through the ear canal. For measurement at the cochlear target, the laser beam was focused on the glass beads on the BM (at the base) and Corti's organ (at the apex) through the cochleostomy in the bulla. The photograph in figure $1 \mathrm{~b}$ shows the direction of the laser beam on the BM at the basal turn and on Corti's organ at the apex.

A hearing laser vibrometer (Polytec HLV 1000, Tustin, Calif., USA) was used to measure the movement of the laser targets at the TM and BM. The amplitude and phase angle of the input sound signals and the velocity of the TM and BM were recorded in a digital signal analyzer (HP35670A) for further analysis. Only data with a total harmonic distortion of less than $10 \%$ of puretone signals according to the distortion index shown on the signal analyzer were accepted. The displacement of the TM or BM was directly calculated from the voltage output of the laser vibrometer velocity decoder.

\section{Results}

\section{Confirmation of $\mathrm{OME}$}

Otoscopic examination of the TMs of all of the LPSinjected ears revealed signs of inflammation. Figure 2 records the findings made in the control and LPS-injected ears on days 3 and 14 after injection. Compared with the 

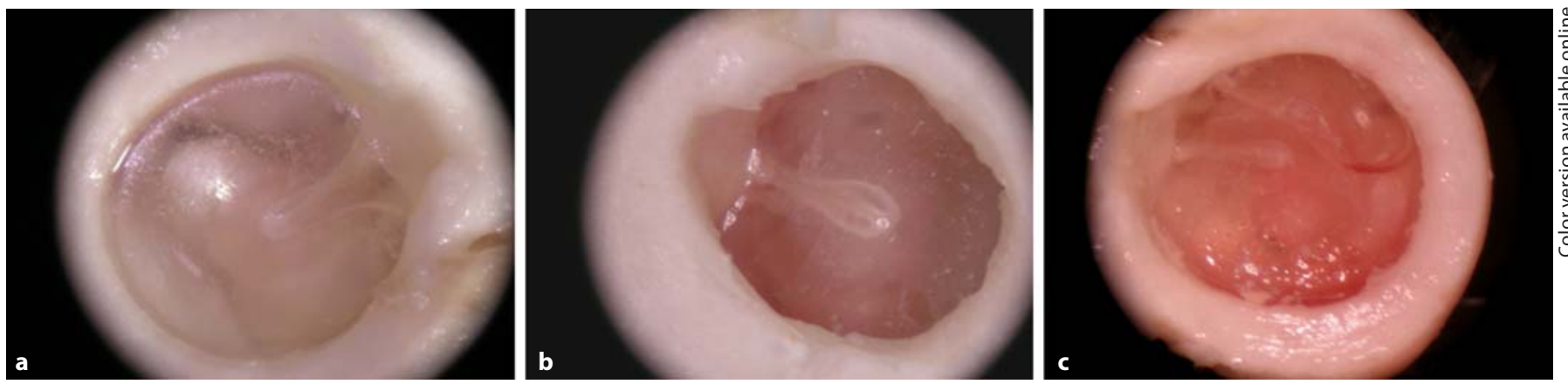

Fig. 2. Photographs of the TM. a A blank control ear. b An OME ear 3 days after LPS injection. c An OME ear 14 days after LPS injection.
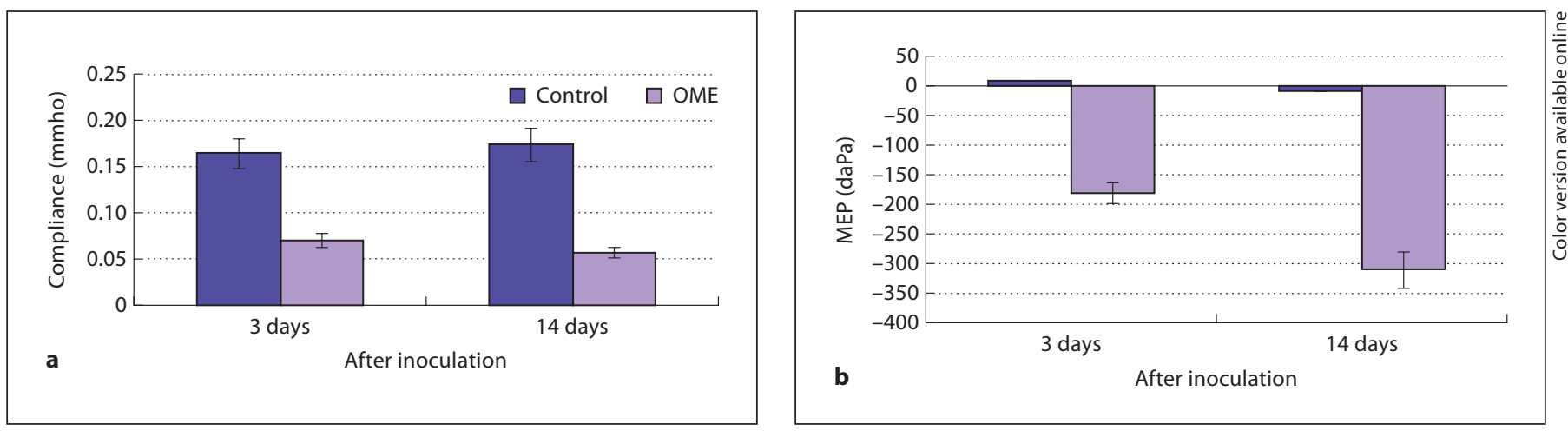

Fig. 3. Data from tympanograms measured in the control and OME ears of two groups (6 in each group) by a high-frequency $(1000 \mathrm{~Hz})$ tympanometer when middle ear pressure was varied from -400 to $400 \mathrm{daPa}$. a Mean compliance data in the control and OME ears. b Mean MEP data in the control and OME ears.

transparent TM of the control ear (fig. 2a), the TMs of the LPS-injected ears were dark and opaque in appearance (fig. 2b, c). The TMs in control ears were transparent, indicating that the inflammation in the LSP-injected ear did not affect the uninjected ear. A middle ear effusion in LPS-injected ears was also observed.

Differences in static middle ear compliance and middle ear pressure (MEP) were observed between the LPSinjected and control ears at the two time points (fig. 3). The mean values of compliance in control ears at 3 and 14 days were $0.16( \pm 0.0125)$ and $0.18( \pm 0.0105)$ mmho, respectively, while the mean values of compliance in LPSinjected ears were 0.07 and $0.06 \mathrm{mmho}$, respectively. The lowest compliance value was measured from the ears tested 14 days after LPS injection. Figure $3 b$ shows the means of MEPs in LPS-injected and control ears at the same times after injection. The mean values of MEP in control ears were close to zero, while the mean values in OME ears were all negative, as $-175( \pm 10.5)$ and $-325( \pm 15.9)$ $\mathrm{daPa}$ for ears at 3 and 14 days, respectively.

Middle ear fluid was observed in all of the LPS-injected ears. The mean amount of effusion in the middle ear was measured at 0.2 and $0.17 \mathrm{ml}$ on 3 and 14 days after injection, respectively. It is noted that the middle ear volume of guinea pig is about $0.2-0.3 \mathrm{ml}$. By visual assessment, the effusion level varied from about half-filled (up to the umbo) to fully filled. All of the control ears had a clear middle ear cavity without signs of inflammation.

These results are similar to those observed previously with LPS middle ear injection [Ohashi et al., 1991]. Our additional tympanometry results give added evidence for successful induction of OME in the experimental ears. All ears injected with LPS at both survival times met the three criteria for OME. 


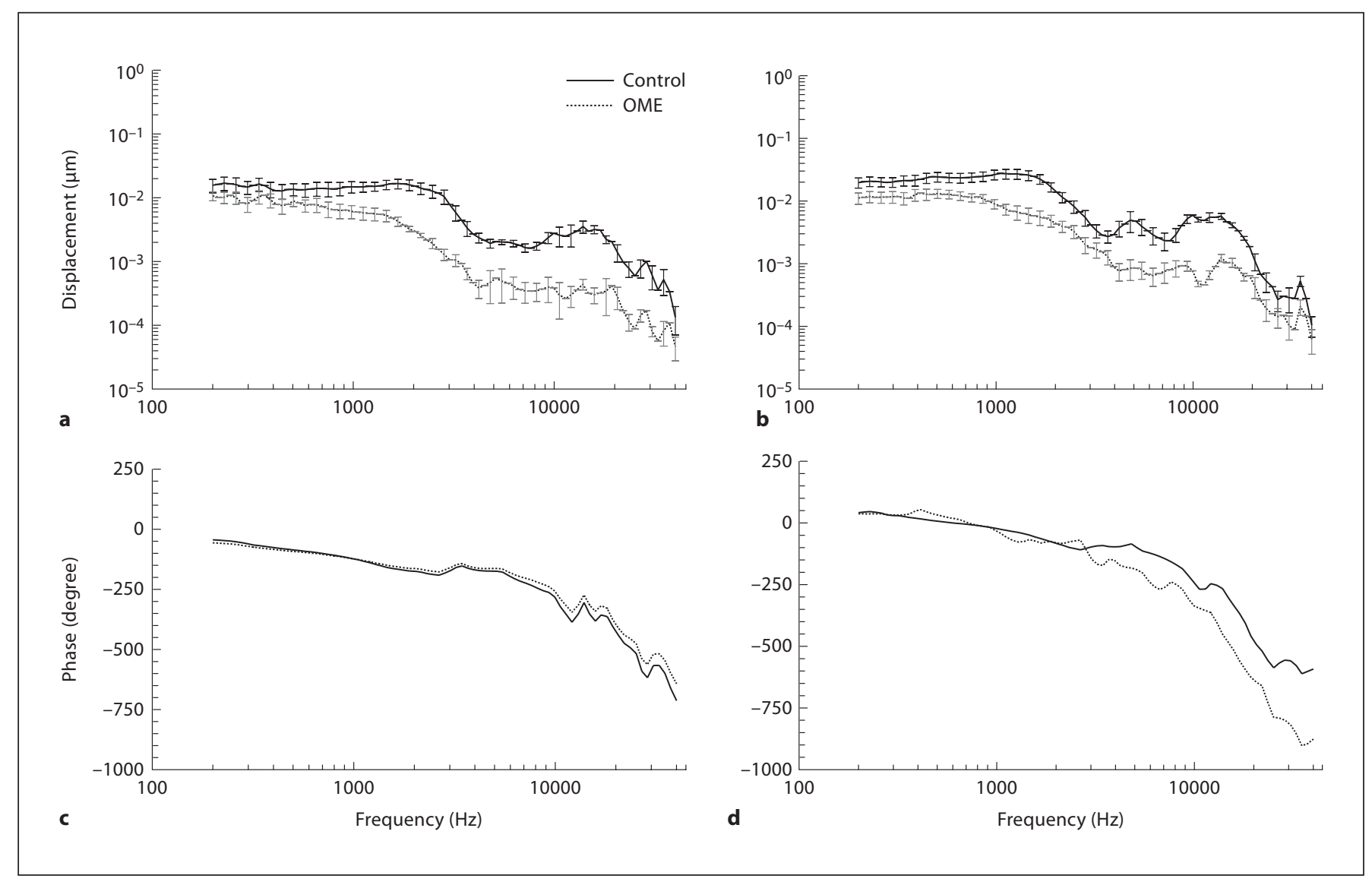

Fig. 4. Mean ( \pm SD) displacement magnitude $(\mathbf{a}, \mathbf{b})$ and phase $(\mathbf{c}, \mathbf{d})$ of the TM in response to $80 \mathrm{~dB} S \mathrm{SL}$ at the external ear canal at frequencies of $(200 \mathrm{~Hz}-40 \mathrm{kHz})$. a, c Group 1 (3 days after LPS inoculation). b, d Group 2 (14 days after receiving inoculation). Error bars are 95\% confidence intervals $(\mathrm{p}<0.05) . \mathrm{n}=6$ in each group. Student's t tests were performed between each experimental curve and the control curve.

\section{Umbo Mobility Change by OME}

The results from blank control ears were very close to the results from contralateral control ears as reported by Dai and Gan [2008]. We also used saline as a control and did the experimental measurement of umbo movements in three types of control: blank, saline, and contralateral controls. We observed that the saline injected into the middle ear cavity was absorbed within 2-3 days and that the vibration measurements of the saline-treated ears were similar to the blank control ears. Therefore, the contralateral control was used as the control in the present study.

Figure 4 shows the mean $( \pm \mathrm{SD})$ spectral displacement magnitude (fig. $4 \mathrm{a}, \mathrm{b}$ ) and phase (fig. $4 \mathrm{c}, \mathrm{d}$ ) of the TM (umbo) vibration measured in OME ears at 3 and 14 days.

As shown in figure 4, TM movement in the OME ear decreased over the frequency range of $200 \mathrm{~Hz}-40 \mathrm{kHz}$ at both survival times. The statistical results of ears collected 3 days after injection show that TM movement was significantly reduced in OME ears at frequencies greater than $250 \mathrm{~Hz}$. Figure $4 \mathrm{~b}$ shows that the displacement of the OME ears collected at 14 days decreased significantly at all frequencies, except for those higher than $20 \mathrm{kHz}$.

\section{Change in BM Movement in OME Ears}

Figure 5 shows the mean ( \pm SD) spectral displacement sensitivity magnitude (fig. 5a, b, displacement divided by stimulus pressure) and phase (fig. $5 c, d$ ) of the $\mathrm{BM}$ at the apex and basal turn measured in OME ears on the 3rd day after inoculation. As shown in figure $5 \mathrm{a}$, the $\mathrm{BM}$ displacement at the apex was high at low frequencies between 200 and $500 \mathrm{~Hz}$ and the maximum displacement sensitivity occurred at around $300 \mathrm{~Hz}$ with a value of about $400 \mathrm{~nm} / \mathrm{Pa}$ in control ears. For the OME ears, the 


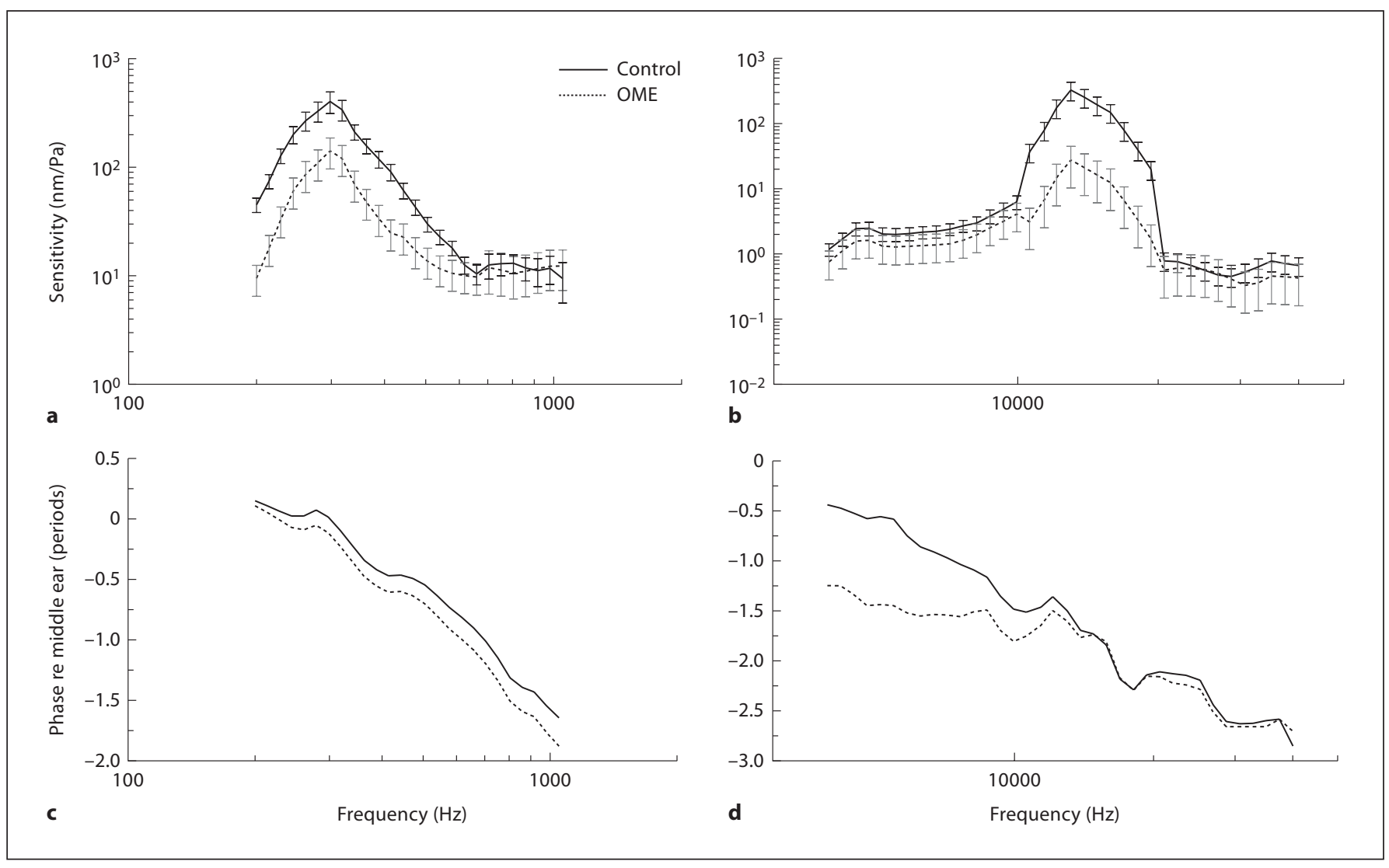

Fig. 5. Mean ( $\pm \mathrm{SD}) \mathrm{BM}$ displacement sensitivity magnitude $(\mathbf{a}, \mathbf{b})$ and phase $(\mathbf{c}, \mathbf{d})$ in response to $80 \mathrm{~dB} S P L$ in the external ear canal at frequencies of $200 \mathrm{~Hz}-40 \mathrm{kHz}$ in group 1 experiments. a, c Apex. b, d Basal turn. Error bars are $95 \%$ confidence intervals $(\mathrm{p}<0.05) . \mathrm{n}=6$ in each group. Student's t tests were performed between each experimental curve and the control curve.

mean displacement sensitivity of the BM decreased significantly at the frequencies of $200-600 \mathrm{~Hz}$ with a maximum value of $38 \mathrm{~nm} / \mathrm{Pa}$. At the basal turn, the peak of $\mathrm{BM}$ displacement occurred at around $13-15 \mathrm{kHz}$ with a maximum value of $325 \mathrm{~nm} / \mathrm{Pa}$ in the control ears. The displacement sensitivity of the BM in OME ears significantly decreased in comparison with the control at high frequencies of $11-18 \mathrm{kHz}$ with a maximum value of 27 $\mathrm{nm} / \mathrm{Pa}$ at around $13 \mathrm{kHz}$.

Figure 6 shows the BM displacement sensitivity measured in ears of group 2 on the 14th day of LPS inoculation. Similar to the results of group 1, BM displacement decreased at both the basal turn and apex. However, BM movement at the apex in OME ears of group 2 did not decrease significantly at $\mathrm{f}<250 \mathrm{~Hz}$. At the basal turn, the reduction in $\mathrm{BM}$ movement is higher ( $25 \mathrm{~dB}$ off) than that in group 1 ( $20 \mathrm{~dB}$ off).
Cochlear Gain with Respect to Middle Ear Movement

To get a rough estimation of cochlear gain as a function of stimulus frequency, BM displacement to TM movement at the umbo was normalized. Figure 7 shows the cochlear gain in decibels at both the basal turn and apex from measurements in group 1 at 3 days after inoculation. Figure 7a shows the cochlear gain at the apex and that the gain was reduced by OME mainly at 200$600 \mathrm{~Hz}$ (functional frequency range of apex) with the maximum reduction of $16 \mathrm{~dB}$ at $400 \mathrm{~Hz}$. Figure $7 \mathrm{~b}$ shows that the cochlear gain at the basal turn was decreased with OME at frequencies of $5-9 \mathrm{kHz}$ and $11-13 \mathrm{kHz}$ with the maximum reduction of $10 \mathrm{~dB}$ at about $14 \mathrm{kHz}$. There was no significant reduction in cochlear gain at frequencies of $9-11 \mathrm{kHz}$.

Figure 8 shows the cochlear gain obtained from the measurements in group 2 after 14 days of inoculation; the results were similar to those of group 1 . At the apex 


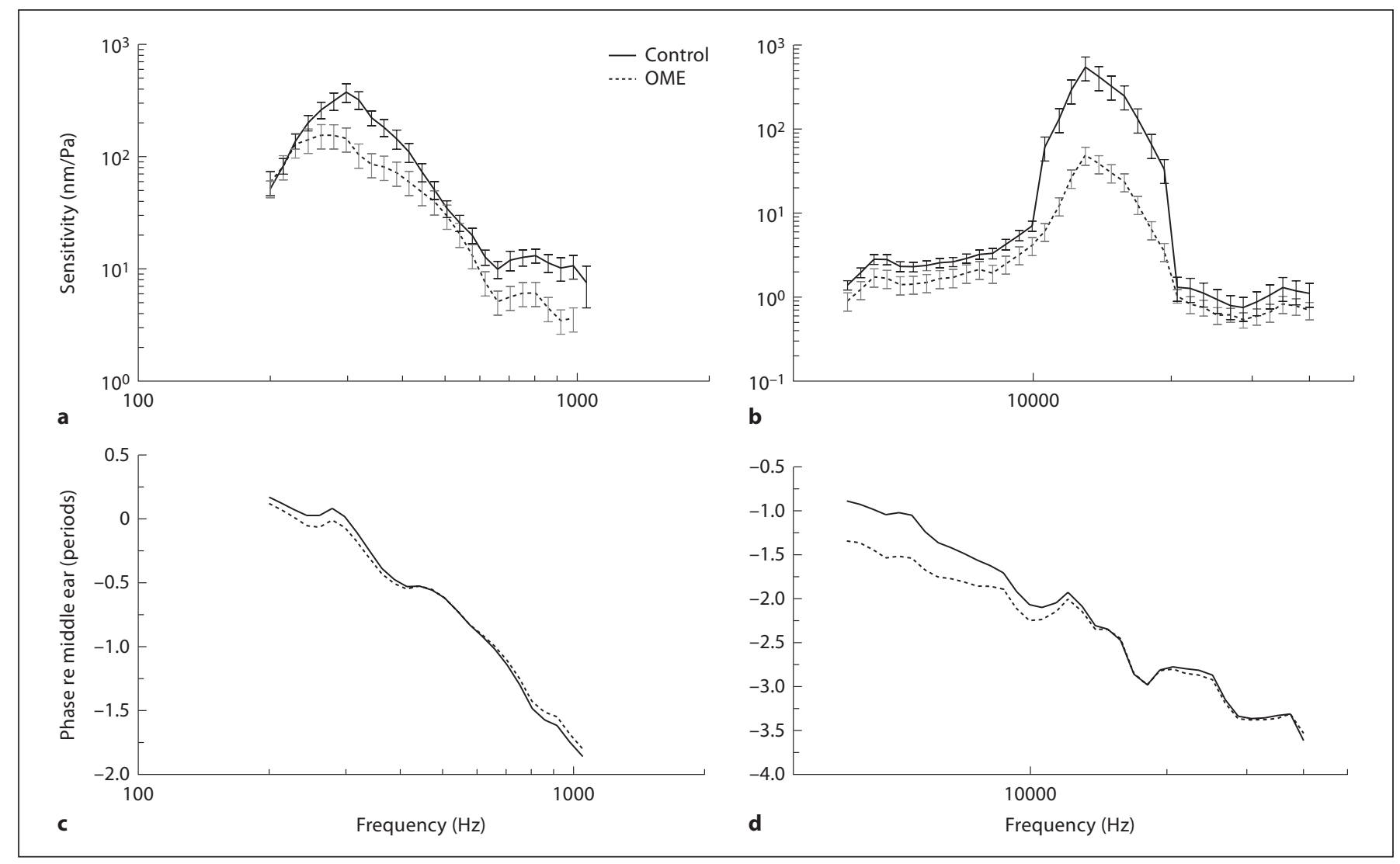

Fig. 6. Mean ( \pm SD) BM displacement sensitivity magnitude $(\mathbf{a}, \mathbf{b})$ and phase $(\mathbf{c}, \mathbf{d})$ in response to $80 \mathrm{~dB}$ SPL in the external ear canal at frequencies of $200 \mathrm{~Hz}-40 \mathrm{kHz}$ in group 2 experiments. a Apex. b Basal turn. Error bars are $95 \%$ confidence intervals $(\mathrm{p}<0.05)$. Student's t tests were performed between each experimental curve and the control curve.
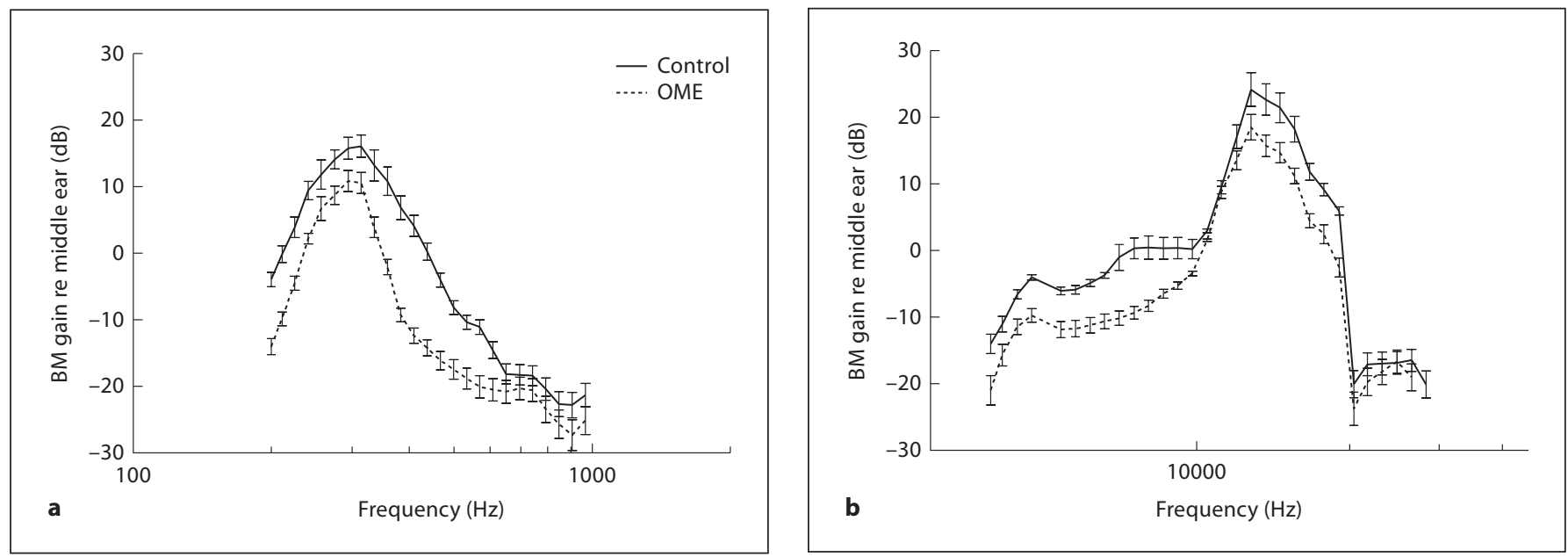

Fig. 7. Cochlear gain with respect to the umbo movement at the apex (a) and basal turn (b) in group 1. 

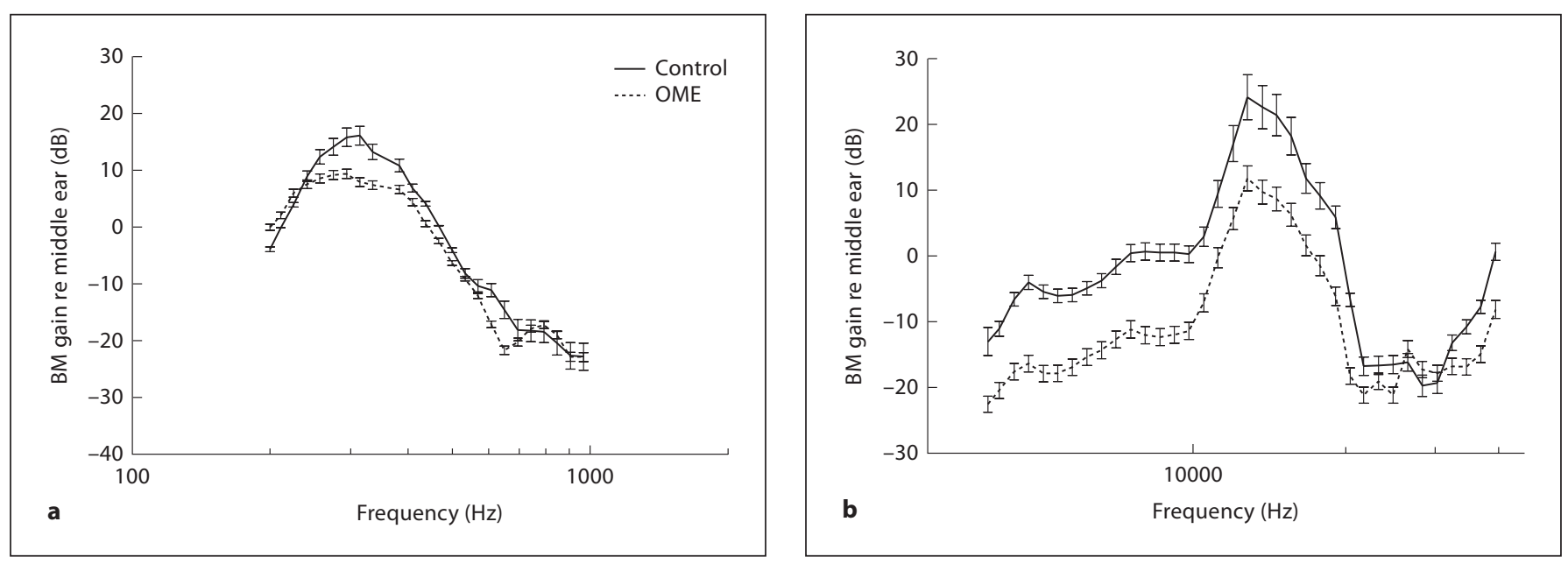

Fig. 8. Cochlear gain with respect to the umbo movement at the apex (a) and basal turn (b) in group 2 .

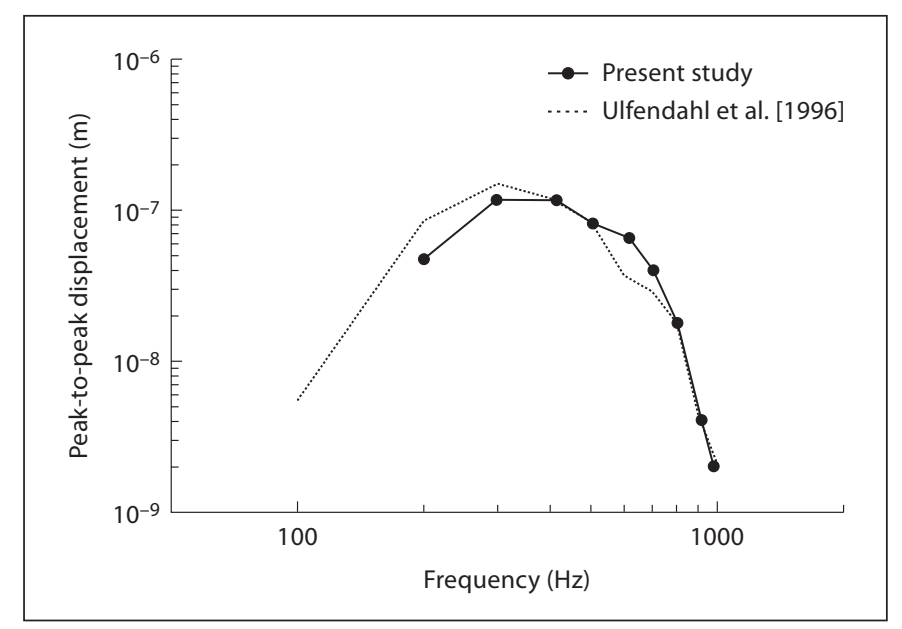

Fig. 9. Comparison of BM vibration amplitude in response to selected intensities at different frequencies [Ulfendahl et al., 1996; fig. 7a, outer hair cell vibration curve].

(fig. 8a), the cochlear gain in OME ears did not show a significant reduction at $200-600 \mathrm{~Hz}$. The maximum reduction in cochlear gain at the apical region was about $7 \mathrm{~dB}$ at around $300 \mathrm{~Hz}$, which is lower than the reduction seen in group 1 . The reduction in cochlear gain at the basal turn of OME ears of group 2 (fig. 8b) was larger than that in group 1 , with a maximum reduction of $13 \mathrm{~dB}$ at around $14 \mathrm{kHz}$.

We also calculated the $\mathrm{Q}_{10}(\mathrm{CF} / \mathrm{BW} 10$, where $\mathrm{BW} 10$ is the bandwidth $10 \mathrm{~dB}$ below the displacement peak (normalized to the umbo displacement) and CF is the char- acteristic frequency or the frequency of the displacement peak to determine the sharpness of cochlear tuning). Table 1 shows the $\mathrm{Q}_{10}$ values in measured ears at the apex and basal turn of the BM in groups 1 and 2. In group 1 (3 days after inoculation), the average control value of $\mathrm{Q}_{10}$ was 1.12 and the mean value in OME ears was 1.01 at the apex. At the basal turn, the mean $\mathrm{Q}_{10}$ value of the control was 5.52 and the mean value of the OME ears was 4.21. The decrease in the $\mathrm{Q}_{10}$ in OME ears at the apex and basal turn was significant (Student's $t$ test, $\mathrm{p}<0.05$ ). Similar decreases of $Q_{10}$ values were seen in OME ears of group 2 after 14 days of inoculation.

\section{Discussion}

\section{Result Summary and Comparison}

In this study, OME was created by injection of LPS in the middle ear of guinea pigs as described previously [Dai and Gan, 2008]. This response is likely due to the release of a variety of vasoactive amines and inflammatory mediators [Musson et al., 1978]. OME was confirmed by morphological observation, low middle ear compliance, and the volume of fluid in the middle ear.

The experiments were performed in two groups, representing early and chronic stages of OME. BM movements were reduced at the basal turn and apex at their characteristic frequencies in OME ears compared with the control in both groups. However, different effects of OME on BM movement were observed between the two stages of OME (fig. 5, 6). In group 1, 3 days after inocula- 
Table 1. List of normalized $\mathrm{Q}_{10}$ values calculated from the BM displacement curves in groups 1 and 2

\begin{tabular}{llllll}
\hline & \multicolumn{2}{l}{3 days (group 1) } & & \multicolumn{2}{l}{ 14 days (group 2) } \\
\cline { 2 - 3 } & apex & basal turn & & apex & basal turn \\
\hline Control & $1.12 \pm 0.037$ & $5.52 \pm 0.240$ & & $1.15 \pm 0.073$ & $5.13 \pm 0.435$ \\
OME & $1.01 \pm 0.041$ & $4.21 \pm 0.320$ & & $1.07 \pm 0.092$ & $4.03 \pm 0.232$ \\
p value & $<0.05$ & $<0.05$ & & $<0.05$ & $<0.05$ \\
\hline
\end{tabular}

The $\mathrm{BM}$ displacement data used for calculation of $\mathrm{Q}_{10}$ were normalized to the umbo movement. $p$ values were calculated by the paired t test between the control and experimental data.

Table 2. Mechanical responses to tones at apical sites of the cochlea

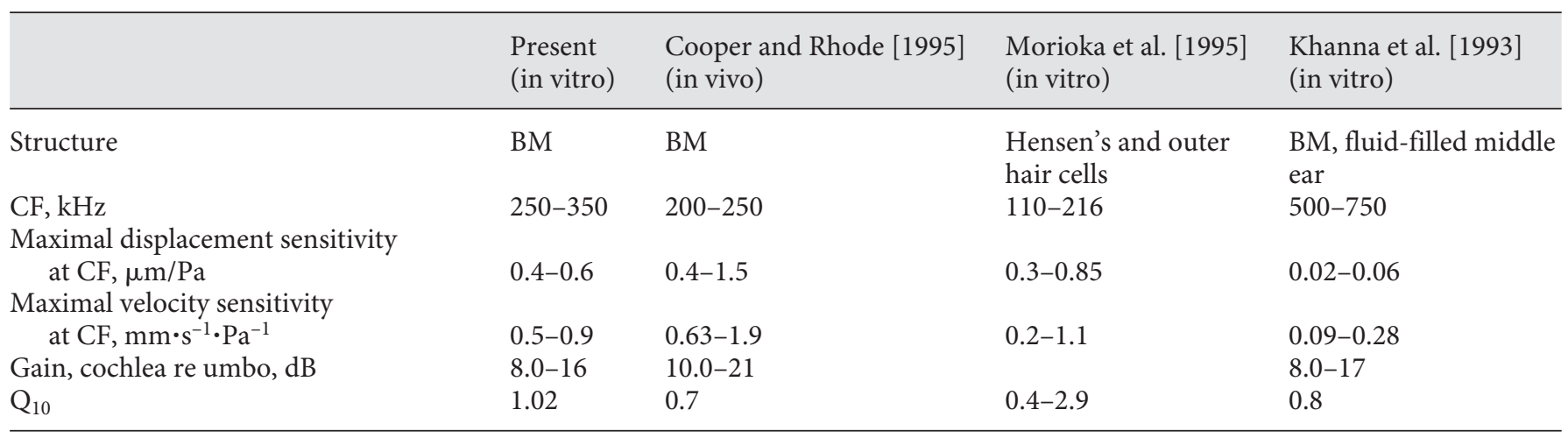

tion, BM movement at the basal turn was reduced mainly at frequencies of $13-17 \mathrm{kHz}$ and the amplitude of the reduction reached $20 \mathrm{~dB}$. At the apex, BM movement was reduced at the range of $200-600 \mathrm{~Hz}$. In group 2, 14 days after inoculation, the reduction in BM movement at the apex occurred at frequencies of $250-400 \mathrm{~Hz}$ and the reduction was less than that in group 1 . The measurements at the basal turn demonstrated that the reduction in $\mathrm{BM}$ displacement sensitivity in group 2 was higher than that in group 1.

The cochlear gain with respect to umbo movement decreased at the apex and basal turn at their characteristic frequencies in both experimental groups. The reductions of cochlear gain demonstrate the changes of cochlear function in OME ears.

Using laser heterodyne interferometry, Ulfendahl et al. [1996] successfully measured the vibration of the BM at the apex in guinea pig temporal bones. Figure 9 shows the comparison of BM displacement at the apex in the present study with the data of Ulfendahl et al. [1996; reproduced from the vibration curve of outer hair cells, fig. 7a]. As seen in figure 9, our measurements in the control ears were similar to their results, corroborating the reliability of measurements in our experimental setup. Also in the current study, the maximum amplitude of BM velocity sensitivity at the basal turn was $0.046 \mu \mathrm{m} / \mathrm{Pa}$, similar to other reports of $0.02-0.06 \mu \mathrm{m} / \mathrm{Pa}$ [Robles and Ruggero, 2001].

Measurement of BM movement at the apex is extremely difficult with the laser vibrometer because of anatomical limitations. Table 2 lists some results of the present and other groups' measurements of the TM. Most of the measurements at the apex were conducted with an in vitro setup. Table 2 shows results from several groups. The characteristic frequencies (CFs) were between 250 and $350 \mathrm{~Hz}$, as can be seen in figures 5 and 6 . The maximum displacement sensitivity of the BM at the apex in our study was $0.4-0.6 \mu \mathrm{m} / \mathrm{Pa}$, which is very comparable with other reports (table 2).

\section{Change in Cochlear Response in OME Ears}

In the peripheral auditory system, vibration of the middle ear serves as the input to the endolymph producing movement in response to the sound stimuli presented in the external ear canal. Fluid accumulation in the middle ear of OME ears is assumed to dampen cochlear 
movement by reducing the movement of umbo or stapes [Ravicz et al., 2004; Gan et al., 2006; Dai et al., 2008; Dai and Gan, 2008]. The results of this study show that vibration of the BM was reduced at the apex and the base in the OME ears. The reduction in BM movement may be due partially to the reduction in movement of the stapes. Huber et al. [2008] tested the influence of rotational motion on cochlear activity and their qualitative results provide supportive evidence that complex movements of the stapes footplate may lead to cochlear activity. Alternatively, the fluid accumulated in the middle ear may also dampen movement of the round window membrane, resulting in increased cochlear impedance and diminished amplitude of BM movement. The mechanism of how the change in stapes motion affects the cochlear response in OME ears needs further study.

Although the round window membrane in humans is much thicker than that in a guinea pig, a water-tight seal is achieved similarly by a single layer of cells: the outer epithelium [Goycoolea and Lundman, 1997; Carpenter et al., 1989; Cureoglu et al., 2005]. The membrane morphology changes due to otitis media [Sahni et al., 1987] are similar to the observations in guinea pig OME models [Cureoglu et al., 2005]. In other words, the guinea pig OME model with a much thinner round window membrane is presumably a reasonable model for the influence of middle ear pathology affecting the human round window. The agents released through the inflammatory process pass through the round window membrane [Goycoolea, 1992] and affect hair cell responses. Substances released in the middle ear can pass through the round window membrane and cause various inner ear sequelae such as labyrinthitis [Cureoglu et al., 2005; Penha and Escada, 2003]. Nordang and Anniko [2001] measured the round window membrane thickness, examined round window membrane morphological changes and measured auditory brainstem responses in exotoxin-treated rats. Their results show that the thickness of round window membrane doubled in comparison with the control and the animal with the most prominent sensorineural hearing loss (SNHL) had the most prominent inflammatory changes. Their results are in agreement with the findings of other groups [Kawauchi et al., 1989; Johansson et al., 1993]. Brookhouser et al. [1979] investigated the relationship between SNHL and middle ear diseases, and their results show that infants and young children with SNHL are at least prone to experience episodes of OME. In the present study, the change in cochlear response may be partially due to the LPS passing through the round window membrane.
The reduction in BM movement at the apex observed in the 3-day group (up to $15 \mathrm{~dB}$, fig. $5 \mathrm{a}$ ) was more than that of the 14-day group (up to $11 \mathrm{~dB}$, fig. $6 \mathrm{a}$ ). The information of change in BM movement at the apex in OME ears is important to compare with the clinical measurements such as tympanometric results, which usually only test low frequencies (e.g. $226 \mathrm{~Hz}$ [Onusko, 2004]). The current study reports for the first time the effect of OME on $\mathrm{BM}$ movement at low frequencies using a guinea pig OME model.

At the basal end of the cochlea, the BM is anatomically close to the round window, within $2 \mathrm{~mm}$. The movement of the BM in this region has been measured in many previous studies [Nuttall et al., 1991; Robles et al., 1986; Sellick et al., 1982; Murugasu and Russell, 1995], but the change in BM movement at the basal turn in OME ears has not been reported. In this study, the movement of the BM at the basal turn in our guinea pig OME model was measured with a laser Doppler vibrometer. Our results demonstrate that the movement of the BM at the basal turn was reduced in the presence of OME in both experimental groups. At the base of the mammalian cochlea, the sharp frequency tuning of auditory nerve fibers is fully determined by the vibration of the BM [Robles and Ruggero, 2001]. However, the sharp tuning largely disappears immediately after death [Robles and Ruggero, 2001; Rhode, 1971], and the change in cochlear response in this study is most likely due to the change in stapes motion.

According to the study of Papp et al. [2003], reduction in BM movement at the base in an in vivo setup can probably be explained by the closer location of the hair cells responsible for high-frequency hearing to the base of the cochlea and to the round window. Harmful agents (like LPS) penetrating the round window membrane may reach these cells in a higher concentration in this way. The different cochlear responses may reflect varied effects of OME on cochlear function at different stages of the middle ear inflammation. The 14-day group may reflect the chronic effect of OME, and it is possible that temporary or permanent changes in the sensorineural thresholds could be observed. This speculation is in agreement with the studies in a pediatric population with OME. Papp et al. [2003] indicate that the incidence of SNHL increased with the duration of middle ear disease.

Variations of $\mathrm{Q}_{10}$ values suggest that the displacement peak of BM movement becomes less sharp in the OME ears in comparison with the control ears. The changes in $\mathrm{Q}_{10}$ that are reported are small and could be affected by a 
change in the frequency dependence of the stapes producing changes in the bandwidth of the middle ear. It is noted that the $\mathrm{Q}_{10}$ of 5.12 at $80 \mathrm{~dB}$ is high (almost the same level as with the in vivo measurement). Two possibilities could account for these high values: first, the measurement was completed within a relatively short time and the hair cells may still have an active physiologic response to sound stimulation; second, the BM displacement used for the calculation of $\mathrm{Q}_{10}$ was normalized to the umbo movement in response to the sound stimulation.

\section{In vitro Measurement}

In vitro preparations of the cochlea have been extensively and successfully used for studying various aspects of the peripheral auditory system [Ashmore, 1987; Reuter and Zenner, 1990; Dallos et al., 1991; Ulfendahl et al., 1996]. The preparation of the guinea pig temporal bone initially met with skepticism regarding its viability and suitability for study of the inner ear. However, it has been demonstrated that the mechanical responses obtained from the cochlea in the isolated temporal bone compare well to mechanical recordings from the cochlea in living animals [Ulfendahl et al., 1996].

The effusion in the middle ear of the live, sedated guinea pig offered limited access to the cochlea, but the isolated temporal bone preparation allows easier access to the cochlea. The main purpose of this study was to investigate the change in $\mathrm{BM}$ movement in the presence of OME. The in vitro preparation of guinea pig temporal bones gave us a suitable setup for measuring BM vibration without losing important mechanical information. The results show a stable and repeatable reduction in BM vibrations in two experimental groups. The key for successful measurement with this model was to perform the experiment within a short time period (i.e. 1 or $2 \mathrm{~h}$ postmortem) to avoid degradation of measurements over time.

The data related to change in hair cell/nerve response in the presence of OME will be helpful in evaluating cochlear mechanics in this disease process. The first effect of OME on cochlear function may be related to an alteration of the function of the cochlear amplifier, which plays an important role in cochlear response to sound transmission. Given that the function of the outer hair cells is a key part of the cochlear amplifier [Lukashkin et al., 2007; Mellado Lagarde et al., 2008], investigating how middle ear inflammation affects the response of outer hair cell is critical to understand the cochlear mechanical change in ears with OME. However, the measurements of hair cell response in the presence of OME requires an in vivo measurement method. In the present study, the measurement was completed within a relative short time after death of the animal and it is unlikely that the cochlear amplifier was still functioning. To understand the effect of OME on cochlear function and the correlation between the change in hair cell/nerve response and the alteration of BM movement in OME ears, we plan to measure BM movement in living guinea pigs in future investigations.

\section{Correlation between Cochlear and Middle Ear Function}

To evaluate cochlear function in OME, BM sensitivity was normalized to the umbo movement and cochlear gain was obtained as a function of sound stimulus frequency. The cochlear gain with respect to the middle ear vibration gave us an estimate of cochlear function in OME in isolation from the middle ear. As shown in figures 7 and 8, the cochlear gain calculated from measurements in both the 3-and 14-day groups was lower with OME compared with those measured from controls. The reduction in cochlear gain is consistent with an effect of OME on cochlear function. It is noted that the maximum gain of $\mathrm{BM}$ response relative to the umbo vibration was around $17 \mathrm{~dB}$ at the apex, and $25 \mathrm{~dB}$ at the base of the cochlea in the controls. These data were lower than those reported by Cooper and Rhode [1992] (about $35 \mathrm{~dB}$ at the pex of chinchillas) and Sellick et al. [1983] (around $55 \mathrm{~dB}$ at the basal turn of guinea pigs). This is likely due to the inherent differences between in vitro and in vivo measurements. Therefore, our measurements comparing treated to untreated ears in vitro provide a meaningful comparison.

The mechanism of reduction in cochlear gain in OME needs further investigation. Several factors could be responsible, including an increase in cochlear impedance and/or cochlear fluid pressure by the inflammatory response. The changes in cochlear gain shows that cochlear function was affected by OME and the cochlear function change in OME in both experimental groups was consistent with the change in TM movement. Those results indicate that the conductive hearing loss associated with $\mathrm{OME}$ is likely related initially to the middle ear function impairment and later to cochlear function variation. Nakajima et al. [2005] found that the flexibility in the ossicular chain due to the joints can affect a loss in sound-induced mechanical energy between the umbo and the stapes, with a concomitant reduction in the sound-induced motion of the stapes. Thus, a better approach to evaluate the co- 
chlear movement changes in OME is to normalize the $\mathrm{BM}$ motion to the stapes or round window motion, which directly drives the cochlear fluid movement. The measurement of round window movement in OME was reported in figure 7 of our early paper [Dai and Gan, 2008]. The change in cochlear function related to variations in middle ear function with OME needs further analysis with round window movement variation.

\section{Conclusion}

The mobility of the BM at the basal turn and apex was measured in a guinea pig OME model with an in vitro experimental setup. The results demonstrate that the displacement sensitivity of the BM was reduced in the pres- ence of OME at characteristic frequencies in comparison with the controls. The reduction in cochlear gain with respect to umbo movement is consistent with a decrease in cochlear function. This study provides useful data for middle ear-cochlear mechanics analysis in OME.

\section{Acknowledgements}

This work was supported by Oklahoma Center for the Advancement of Science \& Technology (HR06-036) and NIH/ NIDCD R01DC006632. The authors thank Don Nakmali at Hough Ear Institute for his expert technical assistance. They thank Dr. Ann Thompson at the University of Oklahoma Health Sciences Center for her many useful suggestions to improve this paper. Three anonymous reviewers are gratefully acknowledged for their great suggestions to improve this paper. We also thank Dr. Mark Wood at Hough Ear Institute for his efforts in editing the paper.

\section{References}

- Ar A, Herman P, Lecain E, Wassef M, Huy PT, Kania RE: Middle ear gas loss in inflammatory conditions: the role of mucosa thickness and blood flow. Respir Physiol Neurobiol 2007;155:167-176.

-Ashmore JF: A fast motile response in guineapig outer hair cells: the cellular basis of the cochlear amplifier. J Physiol (Lond) 1987; 388:323-347.

Brookhouser PE, Hixson PK, Matkin ND: Early childhood language delay: the otolaryngologist's perspective. Laryngoscope 1979;89: 1898-1913.

Carpenter AM, Muchow D, Goycoolea MV: Ultrastructural studies of the human round window membrane. Arch Otolaryngol Head Neck Surg 1989;115:585-590.

Cheatham MA, Dallos P: Comparison of lowside and high-side two-tone suppression in inner hair cell and organ of Corti responses. Hear Res 1990;50:193-210.

Cody A, Johnstone B: Single auditory neuron response during acute acoustic trauma. Hear Res 1980;3:3-16.

Cooper NP, Rhode WS: Basilar membrane mechanics in the hook region of cat and guineapig cochlea: sharp tuning and nonlinearity in the absence of baseline position shifts. Hear Res 1992;63:163-190.

-Cooper NP, Rhode WS: Nonlinear mechanics at the apex of the guinea-pig cochlea. Hear Res 1995;82:225-243.

Cureoglu S, Schachern PA, Rinaldo A, Tsuprun V, Ferlito A, Paparella MM: Round window membrane and labyrinthine pathological changes: an overview. Acta Otolaryngol 2005;125:9-15.
Dai C, Gan RZ: Change of middle ear transfer function in otitis media with effusion model of guinea pigs. Hear Res 2008;243:78-86.

Dai C, Wood MW, Gan RZ: Tympanometry and laser Doppler interferometry measurements on otitis media with effusion model in human temporal bones. Otol Neurotol 2007;28: 551-558.

Dai C, Wood MW, Gan RZ: Combined effect of fluid and pressure on middle ear function. Hear Res 2008;236:22-32.

Dallos P, Evans BN, Hallworth R: Nature of the motor element in electrokinetic shape chang es of cochlear outer hair cells. Nature 1991; 350:155-157.

Gan RZ, Dai C, Wood MW: Laser interferometry measurements of middle ear fluid and pressure effects on sound transmission. J Acoust Soc Am 2006;120:3799-3810.

Goycoolea MV: The round window membrane under normal and pathological conditions. Acta Otolaryngol suppl 1992;493:43-55.

Goycoolea MV: Clinical aspects of round window membrane permeability under normal and pathological conditions. Acta Otolaryngol 2001;121:437-447.

Goycoolea MV, Lundman L: Round window membrane. Structure function and permeability: a review. Microsc Res Tech 1997;36: 201-211.

Gummer AW, Hemmert W, Morioka I, Reis P, Reuter G, Zinner HP: Cellular motility measured in the guinea-pig cochlea; in Duifhuis H, Horst JW, Van Dijk P, and van Netten SM (eds): Biophysics of Hair Cell Sensory Systems. Singapore, World Scientific 1993, pp 229-239.
Hemmert W, Zinner HP, Gummer AW: Characteristics of the travelling wave in the low-frequency region of a temporal-bone preparation of the guinea-pig cochlea. Hear Res 2000;142:184-202.

-Huber AM, Sequeira D, Breuninger C, Eiber A: The effects of complex stapes motion on the response of the cochlea. Otol Neurotol 2008; 29:1187-1192.

International Team for Ear Research: Cellular vibration and motility in the organ of Corti. Acta Otolaryngol Suppl 1989;467:1-279.

Johansson U, Hellström S, Anniko M: Round window membrane in serous and purulent otitis media. Structural study in the rat. Ann Otol Rhinol Laryngol 1993;102:227-235.

Kawauchi H, DeMaria TF, Lim DJ: Endotoxin permeability through the round window. Acta Otolaryngol Suppl 1989;57:100-115.

Khanna SM, Keilson SE, Ulfendahl M, Teich MC: Spontaneous cellular vibrations in the guinea-pig temporal-bone preparation. Br J Audiol 1993;27:79-83.

Lukashkin AN, Walling MN, Russell IJ: Power amplification in the mammalian cochlea Curr Biol 2007; 17:1340-1344

Lynch TJ 3rd, Nedzelnitsky V, Peake WT: Input impedance of the cochlea in cat. J Acoust Soc Am 1982;72:108-130.

Mellado Lagarde MM, Drexl M, Lukashkina VA, Lukashkin AN, Russell IJ: Outer hair cell somatic, not hair bundle, motility is the basis of the cochlear amplifier. Nat Neurosci 2008; 11:746-748.

Morioka I, Reuter G, Reiss P, Gummer AW, Hemmert W, Zinner HP: Sound-induced displacement responses in the plane of the organ of Corti in the isolated guinea-pig cochlea. Hear Res 1995;83:142-150. 
Murugasu E, Russell I: Salicylate ototoxicity: the effects on basilar membrane displacement, cochlear microphonics, and neural responses in the basal turn of the guinea pig cochlea. Auditory Neurosci 1995;1:139-150.

-Musson RA, Morrison DC, Ulevitch RJ: Distribution of endotoxin (lipopolysaccharide) in the tissues of lipopolysaccharide-responsive and -unresponsive mice. Infect Immun 1978; 21:448-457.

- Nakajima HH, Ravicz ME, Merchant SN, Peake WT, Rosowski JJ: Experimental ossicular fixations and the middle ear's response to sound: evidence for a flexible ossicular chain. Hear Res 2005;204:60-77.

-Nordang L, Anniko M: Hearing loss in relation to round window membrane morphology in experimental chronic otitis media. ORL J Otorhinolaryngol Relat Spec 2001;63:333340 .

Nuttall AL, Dolan DF, Avinash G: Laser Doppler velocimetry of basilar membrane vibration. Hear Res 1991;51:203-213.

Ohashi Y, Nakai Y, Esaki Y, Ohno Y, Sugiura Y, Okamoto H: Experimental otitis media with effusion induced by lipopolysaccharide from Klebsiella pneumoniae. Mucociliary pathology of the eustachian tube. Acta Otolaryngol suppl 1991;486:105-115.
Onusko E: Tympanometry. Am Fam Physician 2004;70:1713-1720.

-Papp Z, Rezes S, Jokay I, Sziklai I: Sensorineural hearing loss in chronic otitis media. Otol Neurotol 2003;24:141-144.

Penha R, Escada P: Interrelations between the middle and inner ear in otitis media. Int Tinnitus J 2003;9:87-91.

Ravicz ME, Rosowski JJ, Merchant SN: Mechanisms of hearing loss resulting from middleear fluid. Hear Res 2004;95:103-130.

Reuter G, Zenner H: Active radial and transverse motile responses of outer hair cells in the organ of Corti. Hear Res 1990;43:219-230.

$\checkmark$ Rhode WS: Observations of the vibration of the basilar membrane in squirrel monkeys using the Mossbauer technique. J Acoust Soc Am 1971;49:1218-1231.

Robles L, Ruggero MA: Mechanics of the mammalian cochlea. Physiol Rev 2001;81:13051352 .
Robles L, Ruggero MA, Rich NC: Basilar membrane mechanics at the base of the chinchilla cochlea. I. Input-output functions, tuning curves, and response phases. J Acoust Soc Am 1986;80:1364-1374.

-Sahni RS, Paparella MM, Schachern PA, Goycoolea MV, Le CT: Thickness of the human round window membrane in different forms of otitis media. Arch Otolaryngol Head Neck Surg 1987;113:630-634.

Sellick PM, Patuzzi R, Johnstone BM: Measurement of basilar membrane motion in the guinea pig using the Mossbauer technique. J Acoust Soc Am 1982;72:131-141.

Sellick PM, Yates GK, Patuzzi R: The influence of Mossbauer source size and position on phase and amplitude measurements of the guinea pig basilar membrane. Hear Res 1983; 10:101-108

Ulfendahl M: Mechanical responses of the mammalian cochlea. Prog Neurobiol 1997;53:331380 .

-Ulfendahl M, Khanna SM, Fridberger A, Flock A, Flock B, Jäger W: Mechanical response characteristics of the hearing organ in the low-frequency regions of the cochlea. J Neurophysiol 1996;76:3850-3862. 